\title{
Ceramide galactosyltransferase (UGT8) as a molecular marker of canine mammary tumor malignancy
}

\author{
Marcin Nowak $^{1}$, Piotr Dziegiel ${ }^{2,3}$, Janusz Madej $^{1}$, Maciej Ugorski ${ }^{4,5}$
}

${ }^{1}$ Department of Pathology, Faculty of Veterinary Medicine, Wroclaw University of Environmental and Life Sciences, Wroclaw

${ }^{2}$ Department of Histology and Embryology, Wroclaw Medical University, Wroclaw

${ }^{3}$ Department of Physiotherapy, Wroclaw University School of Physical Education, Wroclaw

${ }^{4}$ Department of Biochemistry, Pharmacology, and Toxicology, Faculty of Veterinary Medicine, Wroclaw University of Environmental and Life Sciences, Wroclaw

${ }^{5}$ Laboratory of Glycobiology and Cell Interactions, Ludwik Hirszfeld Institute of Immunology and Experimental Therapy Polish Academy of Sciences, Wroclaw, Poland

\begin{abstract}
Thirty-two canine mammary tubulopapillary carcinomas and 14 simple adenomas were studied by immunohistochemistry for the expression of UDP-galactose:ceramide galactosyltransferase (UGT8). The majority of tissue specimens $(57 \%)$ representing adenomas had no or weak reaction with anti-UGT8 antibodies $(0-2$ pts according to IRS scale) in comparison to the majority of carcinomas (90\%) which stained with high intensities (3-9 pts according to IRS scale). When the average values of the reaction intensities (IRS) for malignant and benign tumors were compared, using the Mann-Whitney $U$-test, significant differences in UGT8 expression between them were found $(P<0.001)$. Mammary tubulopapillary carcinomas were further analyzed by IHC and the same rabbit polyclonal antibody directed against UGT8 according to their malignancy grade. It was found that the level of UGT8 increased in tumor specimens together with their grading. A comparison of the average values of the reaction intensity (IRS scale) revealed a significant difference (Mann-Whitney $U$-test, $P<0.05$ ) in UGT8 expression between tumors representing malignancy grades G3 and G1. Based on the obtained results, it is proposed that UGT8 is associated with malignancy of canine mammary gland cells and may have a potential value as a diagnostic marker. (Folia Histochemica et Cytobiologica 2013, Vol. 51, No. 2, 164-167)
\end{abstract}

Key words: UGT8, dogs, tumor markers, mammary gland, tumor

\section{Introduction}

UDP-galactose:ceramide galactosyltransferase (UGT8, CGT, C.E. 2.4.2.62) is the enzyme involved in the synthesis of galactosylceramide (GalCer) which is the major glycosphingolipid of myelin produced by

\footnotetext{
Correspondence address: M. Nowak, Department of Pathology, Faculty of Veterinary Medicine, Wroclaw University of Environmental and Life Sciences; Norwida St. 31, 50-375 Wroclaw, Poland; tel./fax: +48 713281567 ;

e-mail: marcin.nowak@up.wroc.pl
}

oligodendrocytes in the central nervous system and Schwann cells in the peripheral nervous system [1-4]. It was suggested that GalCer is involved in myelin function and stability but not in its biogenesis [5-7]. This glycosphingolpid is also present in other organs such as the kidney, small intestine, colon, liver, and testis [8-11].

It has been shown recently that UGT8 is one of six genes which an elevated level correlates with a highly increased risk of lung metastases in breast cancer patients [12]. Also, elevated expression of UGT8 gene in breast cancer was significantly associated with negative expression of estrogen receptors 
Table 1. Semiquantitative IRS scale taking into account both the percentage of positive cells (A) and the intensity of the reaction color $(\mathrm{B})$, with the final score representing the product of the two variables $(\mathrm{A} \times \mathrm{B})$

\begin{tabular}{|c|c|}
\hline \multicolumn{2}{|l|}{ Point score A B } \\
\hline 0 No cells with positive reaction & No color reaction \\
\hline $1 \leq 10 \%$ Cells with positive reaction & Low intensity of color reaction \\
\hline $211-50 \%$ Cells with positive reaction & Average intensity of color reaction \\
\hline $351-80 \%$ Cells with positive reaction & Intense color reaction \\
\hline $4>80 \%$ Cells with positive reaction & \\
\hline
\end{tabular}

(ER-negativity), and therefore with a more malignant phenotype $[13,14]$. Studies performed on the level of mRNA expression using microarray analysis and quantitative RT-PCR were further confirmed by immunohistochemistry. It has been shown that high expression of UGT8 protein is a significant index of tumor aggressiveness and a potential marker for the prognostic evaluation of lung metastases in breast cancer [15]. So far, there were no reports about the expression of UGT8 in canine mammary gland tumors, therefore, the present study was undertaken to analyses the expression of this enzyme in malignant and benign canine tumors.

\section{Material and methods}

Tissue specimens. Tissue specimens were obtained from 46 female dogs of different pure and mixed breeds, aged 7 to 15 years, undergoing surgery at the Department of Surgery, Faculty of Veterinary Medicine, University of Environmental and Life Sciences, Wroclaw, Poland. The tumors were removed with a margin of approximately $2 \mathrm{~cm}$ of healthy tissues.

Histopathological examination and immunohistochemical reactions (IHC). For histological examination the tumor specimens were fixed in $10 \%$ neutral-buffer $(7.0-7.4 \mathrm{pH})$ formalin and embedded in paraffin. Paraffin sections, mounted on Superfrost slides were dehydrated and stained with hematoxylin and eosin. The tumors were classified on the basis of the diagnostic criteria of the World Health Organization [16]. Malignant tumors were classified according to the Bloom-Richardson scale modified by Elston and Ellis [17]. The method for the evaluation of the malignancy grade included three parameters scored in the scale from 0 to 3 points: formation of tubules (evident, moderate, slight), polymorphism of cell nuclei (slight, moderate, marked), number of mitotic figures per 10 microscope fields at the magnification of $\times 400(0-7,8-16, \geq 17)$. The sum of the points provided potential to distinguish 3 grades of malignancy (G) among the tumors: 0-5 pts. - G1 grade, 6-7 pts. - G2 grade, $8-9$ pts. - G3 grade. Immunohistochemical staining was performed on $4-\mu \mathrm{m}$-thick paraffin sections. First, the sections were deparaffinized in xylene and gradually rehydrated using ethanol. Antigen retrieval was performed by boiling the tissue sections in Antigen Retrieval Solution ( $\mathrm{pH}$ 9, $97^{\circ} \mathrm{C}, 20 \mathrm{~min}$; Dako, Glostrup, Denmark). Endogenous peroxidase activity was blocked by $5 \mathrm{~min}$. incubation with $3 \% \mathrm{H}_{2} \mathrm{O}_{2}$. Then the sections were incubated with a primary rabbit polyclonal antibody directed against UGT8 (Atlas Prestige Antibodies, Stockholm, Sweden) diluted in Background Reducing Antibody Diluent (Dako) for $1 \mathrm{~h}$ at room temperature. Peroxidase conjugated goat secondary antibodies (EnVision/HRP; Dako) and diaminobenzidine tetrachloride (DAB; Dako) were used to visualize the studied antigen. All tissue sections were counter-stained with Mayer's hematoxylin. As positive control for the UGT8 IHC a previously breast cancer specimen characterized by its high expression in cancer cells was utilized, whereas Primary Negative Control (Dako) was used as the negative control [15].

Evaluation of IHC reactions. The IHC sections were evaluated using BX-53 light microscope (Olympus, Tokyo, Japan) by two independent pathologists. The level of UGT8 expression was ranked using the modified semi-quantitative Immunoreactive Remmele Score (IRS) according to Remmele and Stegner (Table 1) [18]. This method takes into account both the proportion of stained cells and the intensity of the reaction, while its final results represent the product of the two parameters, with values ranging from 0 to 12 points (no reaction $=0$ points, weak reaction $=1-$ -2 points, moderate reaction $=3-4$ points and intense reaction $=6-12$ points).

Statistical analysis. When groups of data were compared, but failed to satisfy the assumptions of the parametric test, the Mann-Whitney $U$-test, the non-parametric equivalent of Student's $t$-test, was used. The results were considered statistically significant when $P<0.05$ in both analysis. Software Statistica 7.1 (STATSoft, Cracow, Poland) was used for all analyses. 


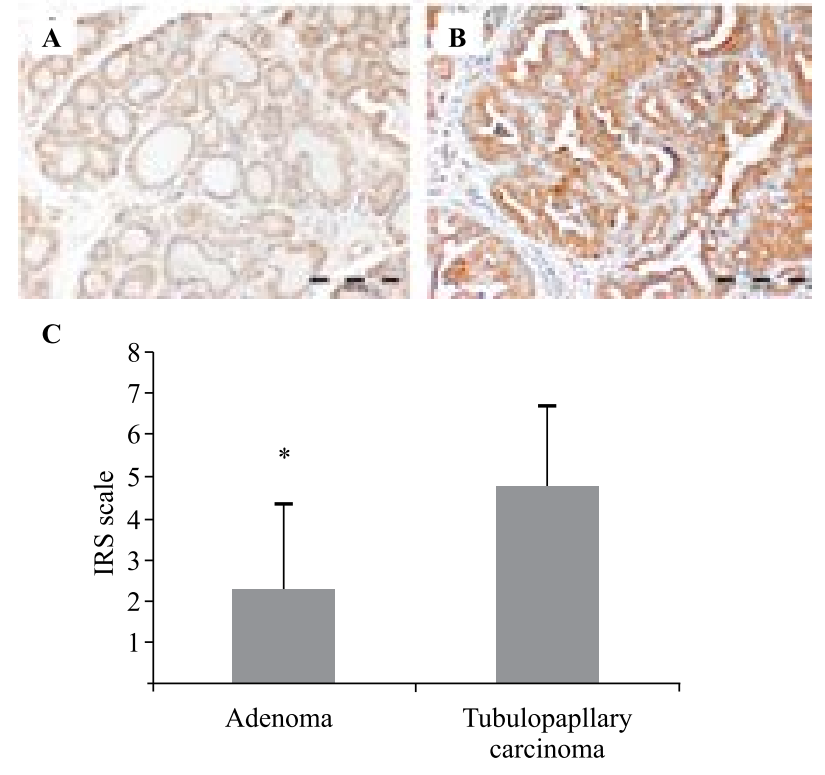

Figure 1. Immunohistochemical staining of (A) simple adenoma and (B) canine mammary tubulopapillary carcinoma with anti-UGT8 rabbit polyclonal antibodies. Reaction intensities with rabbit polyclonal antibodies for UGT8 (C) were calculated on the basis of the semiquantitative IRS scale of Remmele and Stegner (1987) and are represented as means: ${ }^{*} P<0001$ for mammary tubulopapillary carcinomas $(n=32)$ as compared with adenomas $(n=14)$ (Mann -Whitney $U$-test)

\section{Results}

On the basis of histological examination, 32 mammary tubulopapillary carcinomas and 14 simple adenomas were included in the present study. The primary carcinomas were further classified according to the Bloom-Richardson modified by Elston and Ellis [17] as G1 (14 cases), G2 (11 cases) and G3 (7 cases).

Expression of UGT8 in the paraffin sections of malignant and benign tumors was analyzed using rabbit polyclonal antibodies directed against human UGT8 (Figure 1A and B). The majority of tissue specimens $(57 \%)$ representing adenomas had no or weak reaction with anti-UGT8 antibodies $(0-2$ pts according to IRS scale) in comparison to the majority of carcinomas $(90 \%)$ which stained with high intensities (3-9 pts according to IRS scale). When the average values of the reaction intensities (IRS) for malignant and benign tumors were compared by the Mann-Whitney $U$-test, significant differences in UGT8 expression between them were found $(P<0.001$, Figure 1C). Mammary tubulopapillary carcinomas were further analyzed by IHC and the same rabbit polyclonal antibody directed against UGT8 according to their malignancy grade. It was found that the level of this antigen increased in tumor specimens

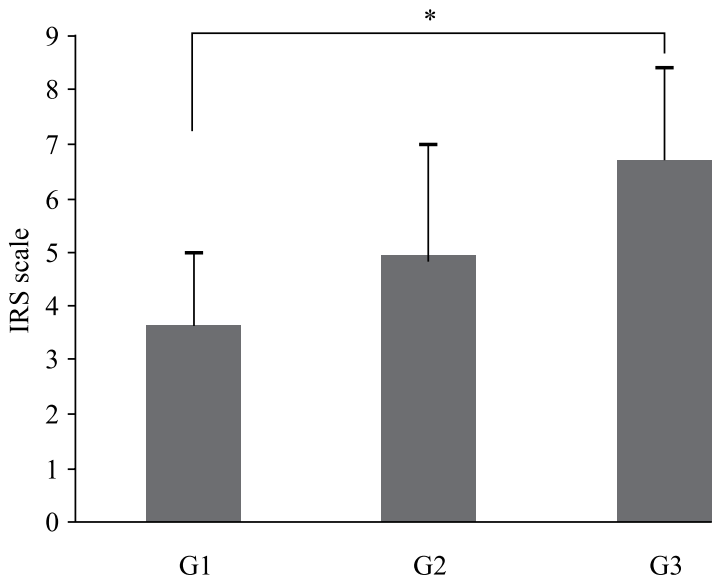

Figure 2. UGT8 expression in canine mammary tubulopapillary carcinomas of different malignancy grades: G1 $(n=14), \mathrm{G} 2(n=11)$ and G3 $(n=7)$. Reaction intensities with rabbit polyclonal antibodies for UGT8 were calculated on the basis of the semiquantitative IRS scale of Remmele and Stegner (1987) and are represented as means:

${ }^{*} P<0.05$ for carcinomas of grade G3 as compared with carcinomas of grade G1 (Mann-Whitney $U$-test)

together with their grading. The average values of the reaction intensity (IRS scale) were significantly different (Mann-Whitney $U$-test, $P<0.05$ ) in UGT8 expression between tumors representing malignancy grades G3 and G1 (Figure 2).

\section{Discussion}

Based on the results obtained for human breast cancer (Dziegiel et al., 2010), the intensities of UGT8 staining in node-positive and node-negative tumors were also compared. Node-positive cases were characterized by a higher average IRS value $(5.25 \pm 1.91)$ than nodenegative cases $(4.45 \pm 2.09)$. However, this difference was not significant (data not shown).

It has been shown recently that expression of UGT8 is highly elevated in breast cancer metastases to the lung in comparison to matched primary tumors and increased expression of this enzyme are associated with progression to a more malignant phenotype [12-15]. Based on these results we studied the expression of UGT8 in canine tubulopapillary carcinomas and simple adenomas. Our results showed that canine malignant tumors had much higher expression of UGT8 in comparison to benign tumors. As in humans, an elevated expression of UGT8 correlated with progression to a more malignant phenotype. Therefore, our data show that UGT8 expression is associated with canine mammary carcinoma and could represent a potentially new marker of malignancy and tumor aggressiveness in dogs. 
The question remains as to the biological meaning of increased expression of UGT8. This enzyme is involved in the biosynthesis of GalCer, and it is possible that in human cancer, including breast cancer, an accumulation of this glycosphingolipid in tumor cells inhibits apoptosis, which facilitates metastatic cells to survive in the hostile micro-environment of the target organ $[15,19]$. Breast tumors and canine mammary neoplasia have many features in common. Both of them are age- and hormone-dependent, and are often similar in their histological appearance as well as the expression of molecular markers [20-23]. Therefore we suggest that GalCer, similarly to humans, may have an antiapoptotic function in canine mammary tumors. However, this hypothesis needs further functional studies.

In summary, our data suggest that UGT8 expression is associated with degree of malignancy of canine mammary gland tumors and may be of potential value as a diagnostic marker.

\section{References}

1. Kapitonov D, Yu RK. Cloning, characterization, and expression of human ceramide galactosyltransferase cDNA. Biochem Biophys Res Commun. 1997;232:449-453.

2. Schulte S, Stoffel W. Ceramide UDP galactosyltransferase from myelinating rat brain: purification, cloning and expression. Proc Natl Acad Sci U.S.A. 1993;90:10265-10269.

3. Sprong H, Kruithof B, Leijendekker R et al. UDP-galactose:ceramide galactosyltransferase is a class I integral membrane protein of the endoplasmic reticulum. J Biol Chem. 1998;273:25880-25888.

4. Marcus J, Popko B. Galactolipids are molecular determinants of myelin development and axo-glial organization. Biochim Biophys Acta. 2002;1573:406-413.

5. Bosio A, Binczek E, Stoffel W. Functional break-down of the lipid bilayer of the myelin membrane in central and peripheral nervous system by disrupted galactosylcerebroside synthesis. Proc Natl Acad Sci U.S.A. 1996;93:13280-13285.

6. Coetzee T, Fujita N, Dupree J et al. Myelination in the absence of galactosylcerebroside and sulfatide: normal structure with abnormal function and regional instability. Cell. 1996;86:209-219.

7. Dupree JL, Girault J, Popko B. Axo-glial interactions regulate the localization of axonal paranodal proteins. J Cell Biol. 1999;147:1145-1151.

8. Kodama S, Igisu H, Siegel D et al. Glycosylceramide synthesis in the developing spinal cord and kidney of the twitcher mouse, an enzymatically authentic model of human Krabbe disease. J Neurochem. 1982;39:1314-1318.
9. Natomi H, Saitoh T, Sugano K et al. Systematic analysis of glycosphingolipids in the human gastrointestinal tract: enrichment of sulfatides with hydroxylated longer-chain fatty acids in the gastric and duodenal mucosa. Lipids. 1993;28:737-742.

10. Nilsson O, Svennerholm L. Characterization and quantitative determination of ganglioside and neutral glycosphingolipids in human liver. J Lipid Res. 1982;23:327-334.

11. Vos JP, Lopes-Cardozo M, Gadella BM. Metabolic and functional aspects of sulfogalactolipids. Biochim Biophys Acta. 1994;1211:125-149.

12. Landemaine T, Jackson A, Bellahcene A et al. A six-gene signature predicting breast cancer lung metastasis. Cancer Res. 2008;68:6092-6099.

13. Ruckhaberle E, Rody A, Engels K et al. Microarry analysis of altered sphingolipid metabolism reveals prognostic significance of sphingosine kinase 1 in breast cancer. Breast Cancer Res Treat. 2008;112:41-52.

14. Yang F, Foekens JA, Yu J, Sieuwerts AM et al. Laser microdissection and microarray analysis of breast tumors reveal ER-alpha related genes and pathways. Oncogene. 2006;25:1413-1419.

15. Dziegiel P, Owczarek T, Plazuk E et al. Ceramide galactosyltransferase (UGT8) is a molecular marker of breast cancer malignancy and lung metastases. Br J Cancer. 2010;103:524-531.

16. Misdorp W, Else RW, Hellmen E et al. Histological classification of the mammary tumors of the dog and the cat. In: World Health Organization International Histological Classification of Tumors of Domestic Animals, ${ }^{\text {nd }}$ series, vol. 7. AFIP, Washington, DC, 1999:1-59

17. Elston CW, Ellis IO. Pathological prognostic factors in breast cancer:experience from a large study with long-term follow -up. Histopathology. 1991;19:403-410.

18. Remmele W, Stegner HE. Vorschlag zur einheitlichen Definition eines immunreaktiven Score (IRS) fuer den immunohistochemischen Ostrogenrezeptor-Nachweis (ER-ICA) im Mammakarzinomgewebe. Pathologe. 1987;8:138-140.

19. Beier UH, Gorogh T. Implications of galactosylcerebrosidase and galactosylcerebroside metabolism in cancer cells. Int $J$ Cancer. 2005;115:6-10.

20. Cotran RS, Kumar V, Robbins SL et al. The breast. In: Pathologic Basis of Disease, ed. Cotran RS, Kumar V, Robbins SL, and Schoen FJ, $5^{\text {th }}$ ed. WB Saunders, Philadelphia, PA, 1994:1089-1111.

21. Schulte S, Stoffel W. Ceramide UDP galactosyltransferase from myelinating rat brain: purification, cloning and expression. Proc Natl Acad Sci. U.S.A. 1993;90:10265-10269.

22. Sprong H, Kruithof B, Leijendekker R et al. UDP-galactose:ceramide galactosyltransferase is a class I integral membrane protein of the endoplasmic reticulum. J Biol Chem. 1998;273:25880-25888.

23. Nowak M, Madej J, Dziegiel P et al. Tumor-associated carbohydrate antigens: sialyl $\mathrm{Le}^{\mathrm{a}}$ and $\mathrm{T} / \mathrm{Tn}$ antigens in canine mammary tumors. Vet Pathol. 2009;46:222-226.

Submitted: 6 February, 2013

Accepted after reviews: 27 May, 2013 